\title{
Fenología reproductiva y anatomía floral de las plantas Aloe vera y Aloe saponaria (Aloaceae) en Cumaná, Venezuela
}

\author{
Róger Velásquez-Arenas ${ }^{1} \&$ José Imery-Buiza ${ }^{2}$ \\ 1. Centro de Investigaciones Ecológicas Guayacán (CIEGUDO). \\ 2. Laboratorio de Genética Vegetal, Departamento de Biología, Hebario IRBR, Universidad de Oriente, Núcleo de Sucre, \\ Cumaná, 6101, Venezuela, Apdo, 245; jimeryb@cantv.net
}

Recibido 04-VIII-2007. Corregido 22-II-2008. Aceptado 31-VII-2008.

\begin{abstract}
Reproductive phenology and flower anatomy of the plants Aloe vera and Aloe saponaria (Aloaceae) in Cumana, Venezuela. The reproductive phenology and the floral anatomy of two species of Aloe kept under nursery conditions were analized in eastern Venezuela from September 2001 to September 2002. $A$. vera flowered between December and May; A. saponaria from November through August. In both species, the anthesis lasted $48 \mathrm{~h}$., the anther dehiscence coincided with the opening of the perianth, the stigma receptivity was higher on the second day of anthesis and nectar production reached $0.34 \mathrm{ml} /$ flower. In A. vera $228 \pm 77$ yellow flowers on long scapes of $76 \pm 11 \mathrm{~cm}$ and with $1-3$ shafts were observed; A. saponaria had $94 \pm 33$ orange flowers on more compact scapes with 3-5 shafts. The pollen/ovule ratio was 4115.2 in $A$. vera and 3 247.1 in A. saponaria, thus they can be classified as compulsory xenogams. Frequent visits of Apis mellifera, Trigona sp., Poliste sp., Eumenes sp., Vespa sp., Leucippus fallax and Amazilia tobaci were recorded. Although both species showed a high production of pollen and nectar, and the presence of potential pollinators, by the end of the flowering period, fruits were only observed in A. saponaria, with a $12 \%$ reproductive efficiency. Coupled with the stigmatic receptivity results and the preliminary intra and interspecific crossing experiments, this suggests the existence of protandry and self-incompatibility as reproductive barriers reducing endogamy in these species. Rev. Biol. Trop. 56 (3): 1109-1125. Epub 2008 September 30.
\end{abstract}

Key words: Reproductive biology, pollination, phenology, floral syndrome, mellitophilia.

El género Aloe, comprende unas 380 especies, distribuidas principalmente en regiones tropicales y subtropicales de África y Asia. En estos ambientes, el género Aloe se diversifica en un amplio espectro que incluye hierbas perennes, arbustos y árboles (Smith y Van-Wyk 1991). La especie $A$. vera (L.) Burm. f. (=A. barbadensis Mill.) se encuentra naturalizada a lo largo de la región del Mediterráneo y zona tórrida del nuevo mundo (Carter 1994). En Venezuela y otros países del Caribe, esta especie es valorada por sus propiedades curativas y uso ornamental.

En general, los estudios preliminares han caracterizado a las plantas de A. vera como hierbas suculentas de uso medicinal, que presenta flores amarillas de $2.5 \mathrm{~cm}$ de largo, agrupadas en racimos individuales o compuestos sobre escapos erguidos de 60 a $100 \mathrm{~cm}$ de alto, perianto tubular, estambres excertos y frutos capsulares dehiscentes, oblongos y triangulares con semillas elipsoidales y aplanadas (Sanabria 1978; Schnee 1984; Hoyos 1985; Sánchez 1999). Las plantas de A. saponaria se han descrito como hierbas medianamente suculentas, de uso ornamental, que se caracterizan por presentar inflorescencias erectas, simples o ramificadas de $60 \mathrm{~cm}$ de alto, con flores colgantes de color anaranjado-escarlata a rojo-escarlata de 3-4 cm de largo, forma tubular y frutos verdosos en forma de cápsulas dehiscentes (Hoyos 1982). 
La escasa información sobre fenología reproductiva y morfología floral de las especies del género Aloe introducidas en América, aunado a la importancia agroindustrial de A. vera y a la necesidad de generar variabilidad genética para mejorar la tolerancia a enfermedades bacterianas, motivaron la realización del presente trabajo con el objetivo de analizar la fenología reproductiva y aspectos anatómicos florales en plantas de $A$. vera y $A$. saponaria, cultivadas en condiciones de vivero en un banco de germoplasma en el oriente de Venezuela.

\section{MATERIAL Y MÉTODOS}

Material vegetal: se utilizaron 50 plantas adultas por especie, cultivadas en macetas plásticas en el vivero del Departamento de Biología de la Universidad de Oriente, ubicado a $10^{\circ} 28^{\prime} 03^{\prime \prime} \mathrm{N}, 64^{\circ} 10^{\prime} 15^{\prime}$ ' W, Cumaná, Venezuela. Las condiciones ambientales de este vivero corresponden a las de un bosque muy seco tropical, intervenido parcialmente para su funcionamiento y con las labores típicas de un centro de conservación ex situ. Las plantas de $A$. vera, provenían originalmente de una población naturalizada a $10^{\circ} 34^{\prime} 21^{\prime \prime} \mathrm{N}$, $64^{\circ} 10^{\prime} 15^{\prime \prime} \mathrm{O}$ en la península de Araya y las de A. saponaria fueron adquiridas en un vivero comercial de la ciudad de Cumaná. Ambas especies se identificaron según Carter (1994), Van-Wyk y Smith (1996).

Descripción de la inflorescencia: la inflorescencia fue estudiada en todas las plantas, comenzando desde la emergencia del escapo. La duración de la inflorescencia se determinó anotando la fecha de apertura de la primera y última flor; el tamaño de la inflorescencia y el escapo se obtuvo mediante la diferencia de longitud desde la base del escapo hasta la primera y última flor abierta. El número de flores por planta se obtuvo contando por intervalos en la inflorescencia y sumando todos los intervalos para obtener el número total de flores por planta. Para la obtención del ancho del escapo, se midió el eje polar y eje ecuatorial, tanto en la base como a la altura de la primera flor abierta, además se describió el color del escapo al momento de iniciarse la floración.

Morfología floral: se recolectaron flores de diez individuos en siete estadios distintos de antesis, desde dos días antes de la antesis $(-2,-1)$, el día de antesis $(0)$, hasta cuatro días después de antesis $(1,2,3,4)$. El análisis morfométrico se realizó midiendo las dimensiones florales (longitud y diámetro del perianto, androceo y gineceo) en material fresco, asimismo, los colores de las distintas piezas florales, se reconocieron por comparación con una escala Prismacolor. La longevidad floral se determinó desde la hora exacta en que ocurría la apertura de la flor (hora de antesis) y la hora en la cual ocurría el cierre floral. En estas flores también se registró la presencia de polen a fin de determinar en qué momento sucedía la dehiscencia de las anteras.

Relación Polen/Óvulo (P/O): se realizaron cortes longitudinales y transversales de ovarios, provenientes de flores en antesis reciente, para determinar el número de óvulos empleando un microscopio estereoscópico. El número de granos de polen se evaluó en flores con anteras maduras, previas al estado de dehiscencia. Estas anteras se colocaron en una cápsula de Petri estéril con una atmósfera húmeda para promover la apertura en condiciones controladas. Una vez abierta, se tomó una antera y se sacudió con un pincel estéril sobre una cápsula milimetrada con glicerina. Se contaron los granos de polen suspendidos en $1 \mathrm{~mm}^{2}$ (tres repeticiones por cápsula) y se determinó el número de granos de polen por flor (NGP) mediante la fórmula NGP $=$ GPUA $\mathrm{x} A C \times$, donde GPUA= promedio del número de granos de polen por unidad de área, $\mathrm{AC}=$ área de la cápsula $\left(\mathrm{mm}^{2}\right), 6=$ número de anteras por flor. La relación polen/óvulo se calculó dividiendo el número de granos polen entre el número de óvulos por flor (Cruden 1977).

Volumen de néctar y concentración de sacarosa: la producción de néctar se midió introduciendo una pipeta Pasteur en el interior 
de la flor hasta extraer todo el néctar, el cual fue depositado en un vial para posteriormente obtener la lectura del volumen con una minipipeta de $1 \mathrm{ml}$. La concentración de sacarosa en el néctar se determinó empleando un refractómetro de mano Atago. Se evaluó una flor por cada estadio de maduración floral en 10 plantas de cada especie. La concentración de azúcar se expresó como el porcentaje masa volumen.

Receptividad del estigma: la receptividad se comprobó empleando el método de Osborn et al. (1988), que consiste en colocar una gota de peróxido de hidrógeno al $40 \%$ sobre los estigmas y tomar como resultado positivo la producción de burbujas. Este ensayo se realizó en diez plantas de cada especie, para cada estadio de maduración de la flor.

Visitantes florales: los visitantes florales fueron estudiados mediante observaciones directas sobre las flores, realizando monitoreos constantes entre las 7:00-10:00 AM y luego por un periodo de $15 \mathrm{~min}$, cada $3 \mathrm{~h}$ durante el resto del día. Estas observaciones fueron llevadas a cabo durante diez días distribuidos en toda la fase de floración. La identificación de las especies visitantes fue realizada, por el personal de los Laboratorios de Zoología y
Entomología del Departamento de Biología de la Universidad de Oriente, mediante fotografías y ejemplares capturados. Durante las observaciones no existían perturbaciones aparentes para la llegada de los visitantes propios de la zona donde se desarrolló la investigación.

Conservación de los especímenes: Una vez finalizadas las evaluaciones reproductivas, se trasladaron las 100 plantas estudiadas hasta el banco de germoplasma de especies suculentas UDO-Biología, Cumaná-Venezuela $\left(10^{\circ} 26^{\prime} 32^{\prime \prime} \mathrm{N}, 64^{\circ} 09^{\prime} 14^{\prime \prime} \mathrm{O}\right)$ y actualmente se mantienen in vivo bajo las condiciones ambientales de un bosque muy seco tropical.

\section{RESULTADOS}

\section{Desarrollo de la inflorescencia de Aloe} vera: en los ejemplares de $A$. vera comenzó la aparición del escapo en diciembre (Fig. 1); el tiempo de desarrollo fue de 16-23 días, presentando una coloración verde-manzana. El período de floración se inició en el mismo mes, extendiéndose hasta mayo, con un pico de floración entre febrero-marzo (Fig. 2), en donde el $81 \%$ de las plantas florecieron. Al culminar este período no hubo formación de frutos en ninguna de las 50 plantas. La inflorescencia en A. vera fue tipo racemosa, con un eje principal

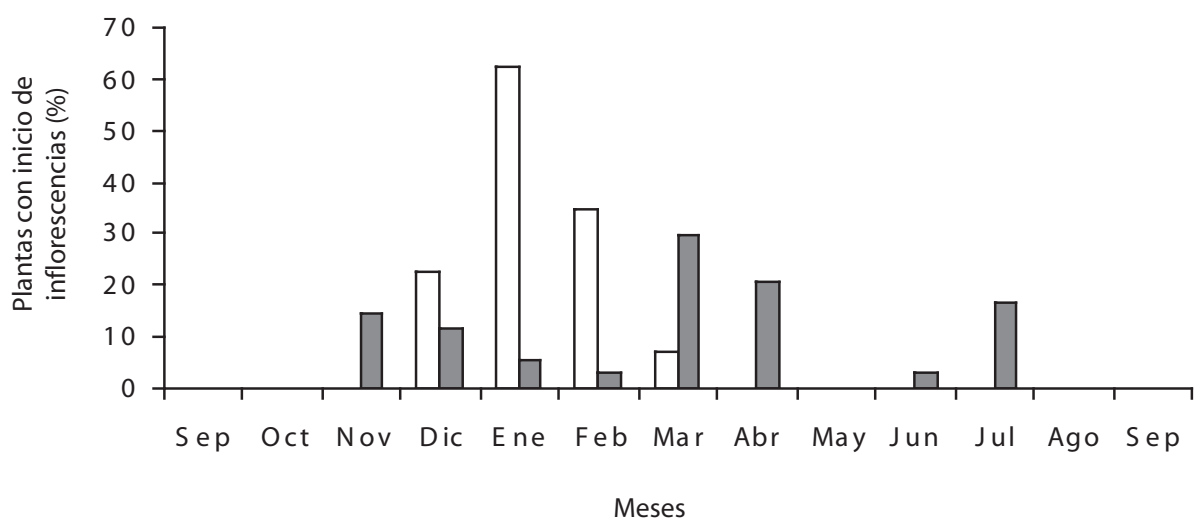

Fig. 1. Emergencia de las inflorescencias en Aloe vera y Aloe saponaria entre septiembre 2001 y septiembre 2002.

Fig. 1. Inflorescence emergency in Aloe vera and Aloe saponaria from September 2001 to September 2002. 


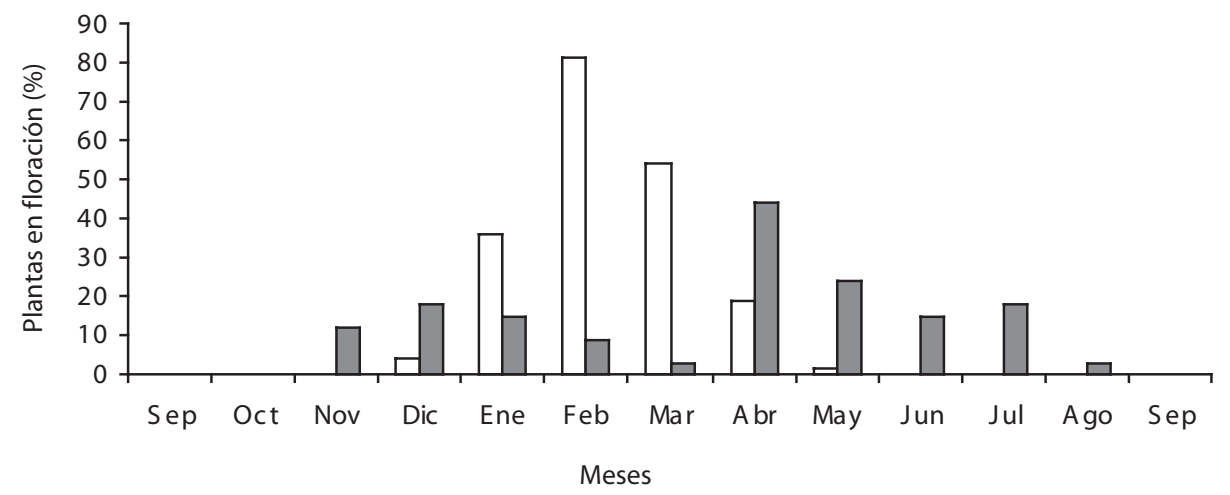

Fig. 2. Floración de Aloe vera y Aloe saponaria entre septiembre 2001 y septiembre 2002.

Fig. 2. Flowering of Aloe vera and Aloe saponaria from September 2001 to September 2002.

y uno a dos ejes secundarios, sobre un escapo erguido, capaz de contener $228.3 \pm 77.3$ flores perfectas. Algunos ejemplares de A. vera presentaron dos a tres inflorescencias durante el periodo de evaluación (Cuadro 1).

Desarrollo de la inflorescencia de Aloe saponaria: se inició con la aparición del escapo en noviembre (Fig. 1), con un tiempo de desarrollo entre 19-21 días y presentando una coloración verde-manzana. El período de floración comenzó en el mismo mes, extendiéndose hasta agosto, con un pico de floración en abril (Fig. 2), donde florecieron $43 \%$ de las plantas. Al culminar este período se observó la formación de $0.12 \pm 0.03$ frutos/flor. El tipo de inflorescencia presente en esta especie fue racemosa compacta simple o ramificada (2-4 ejes secundarios), sobre un escapo ondulado, capaz de contener 93.6 \pm 33.1 flores. Estas plantas pueden presentar dos a cuatro inflorescencias durante su época de floración (Cuadro 1). En ambas especies, la duración de la antesis fue de $48 \mathrm{~h}$.

Morfología floral de Aloe vera y Aloe saponaria: las flores de $A$. vera poseen una coloración amarillo-limón con líneas verdemanzana, colores que cambian a amarillo-ocre a medida que transcurren los distintos estadios de maduración (Fig. 3, Cuadro 2). Cada flor posee una longitud que varía según los estadios, entre 26-34 mm (Cuadro 3), presentando un perianto de forma tubular, ligeramente abultado en la zona intermedia (Fig. 3).

Las flores de $A$. saponaria poseen una coloración rojo-ladrillo con líneas verdes y el ápice de los tépalos de color arena; colores que, a medida que transcurren los distintos estadios de maduración, cambian a rosado con líneas grises claras (Cuadro 4). Estas flores poseen una longitud entre $28-35 \mathrm{~mm}$, según el estadio en que se encuentre (Cuadro 5) y presenta un perianto de forma tubular, con abultamiento en la región subapical y una constricción hacia la base, por encima del ovario (Fig. 3).

Tanto las flores de $A$. vera como las de A. saponaria se encuentran orientadas hacia abajo durante la floración, son bisexuales, actinomorfas y compuestas por seis tépalos unidos desde la base hasta pasada la mitad del perianto (gamotépalos). El androceo está formado por seis estambres que alcanzan una longitud entre $27-32 \mathrm{~mm}$ en $A$. saponaria y 25-35 $\mathrm{mm}$ en $A$. vera (Cuadro 3 y 5); filamentos simples aplanados; anteras oblongas, ditecas, basifijas, con dehiscencia longitudinal, introsas. El gineceo de A. saponaria es de $25-40 \mathrm{~mm}$ de longitud; mientras que en 


\section{CUADRO 1}

Características de las inflorescencias en Aloe vera $y$ A. saponaria

TABLE 1

Inflorescence characteristics in Aloe vera and A saponaria

Carácter

Número de inflorescencias/planta

Número de ejes/inflorescencia

Número de flores en el eje principal

Número de flores en el primer eje secundario

Número de flores en el segundo eje secundario

Número de flores en el tercer eje secundario

Número de flores en el cuarto eje secundario

Número total de flores/inflorescencia

Duración (días) de la floración/inflorescencia

Duración (días) de la inflorescencia

Longitud $(\mathrm{cm})$ del eje de la inflorescencia hasta la primera flor abierta

Longitud (cm) del eje de la inflorescencia hasta la última flor abierta

Longitud $(\mathrm{cm})$ efectiva de la inflorescencia

Ancho de la parte basal del escapo de la inflorescencia

Ancho del escapo a la altura de la primera flor
A. vera

$1.39 \pm 0.52$

$1.67 \pm 0.61$

$167.28 \pm 34.79$

$91.77 \pm 39.11$

$89.80 \pm 12.48$

$20.67 \pm 7.49 *$

$18.00 \pm 7.72$

$16.00 \pm 14.00$

$228.32 \pm 77.29$

$93.58 \pm 33.07^{*}$

$16.42 \pm 5.03$

$14.71 \pm 2.46$

$39.67 \pm 4.91$

$33.58 \pm 6.34 *$

$49.83 \pm 7.73$

$52.53 \pm 11.20^{*}$

$75.67 \pm 11.34$

$62.45 \pm 5.30^{*}$

$25.84 \pm 5.77$

$9.92 \pm 11.43 *$

$15.02 \pm 1.94$

$7.71 \pm 0.95^{*}$

$9.18 \pm 0.85$

Los valores indican promedios \pm desviación estándar. *: diferencias significativas $\left(t_{s} p \leq 0.05\right)$.

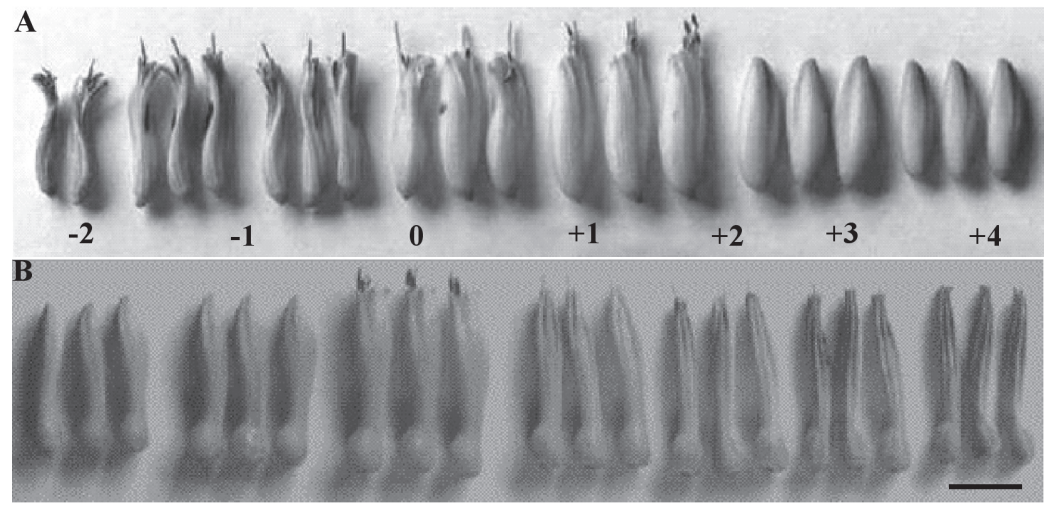

Fig. 3. Morfología de las flores de Aloe vera (A) y A. saponaria (B) en los diferentes estadio de madurez. Los números indican los días antes $(-2,-1)$ y después $(+1,+2,+3,+4)$ de antesis $(0)$. Barra $=1.5 \mathrm{~cm}$.

Fig. 3. Floral morphology from Aloe vera (A) and A. saponaria (B) at different maturity stages. Numbers show days before $(-1,-2)$, and after $(+1,+2,+3,+4)$ anthesis. Bar $=1.5 \mathrm{~cm}$. 


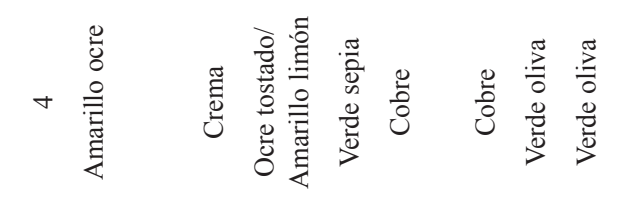

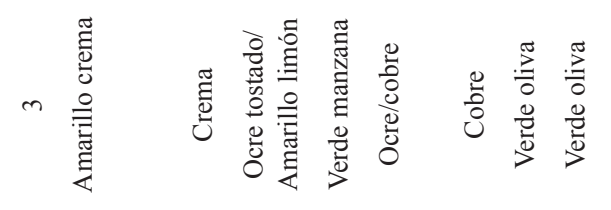

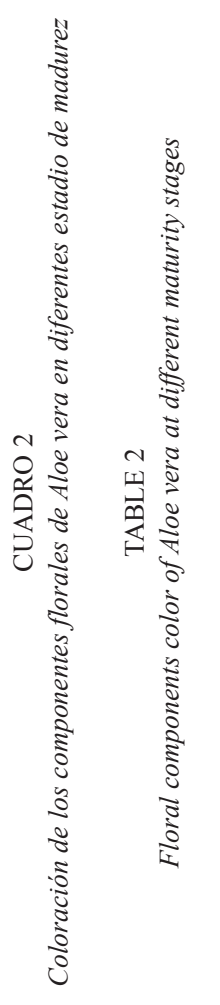

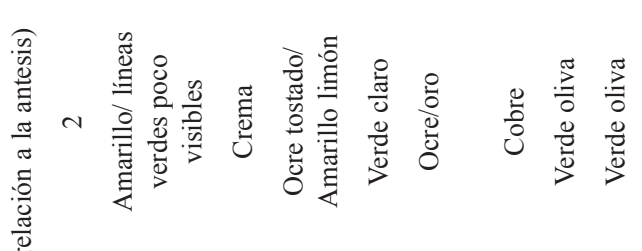

$$
\begin{aligned}
& \text { In!ll }
\end{aligned}
$$

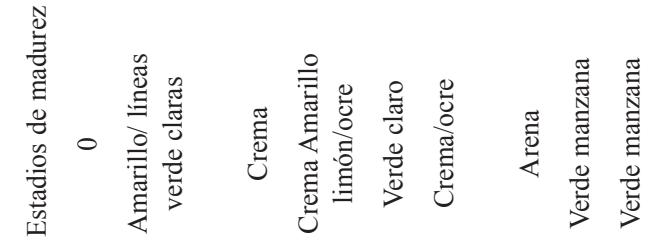

$$
\begin{aligned}
& \text { - In!ly }
\end{aligned}
$$

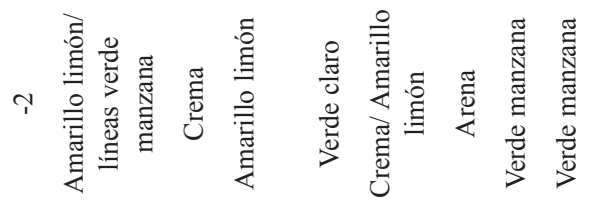

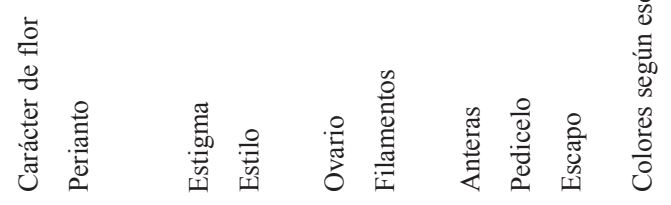




$$
\text { - menn }
$$

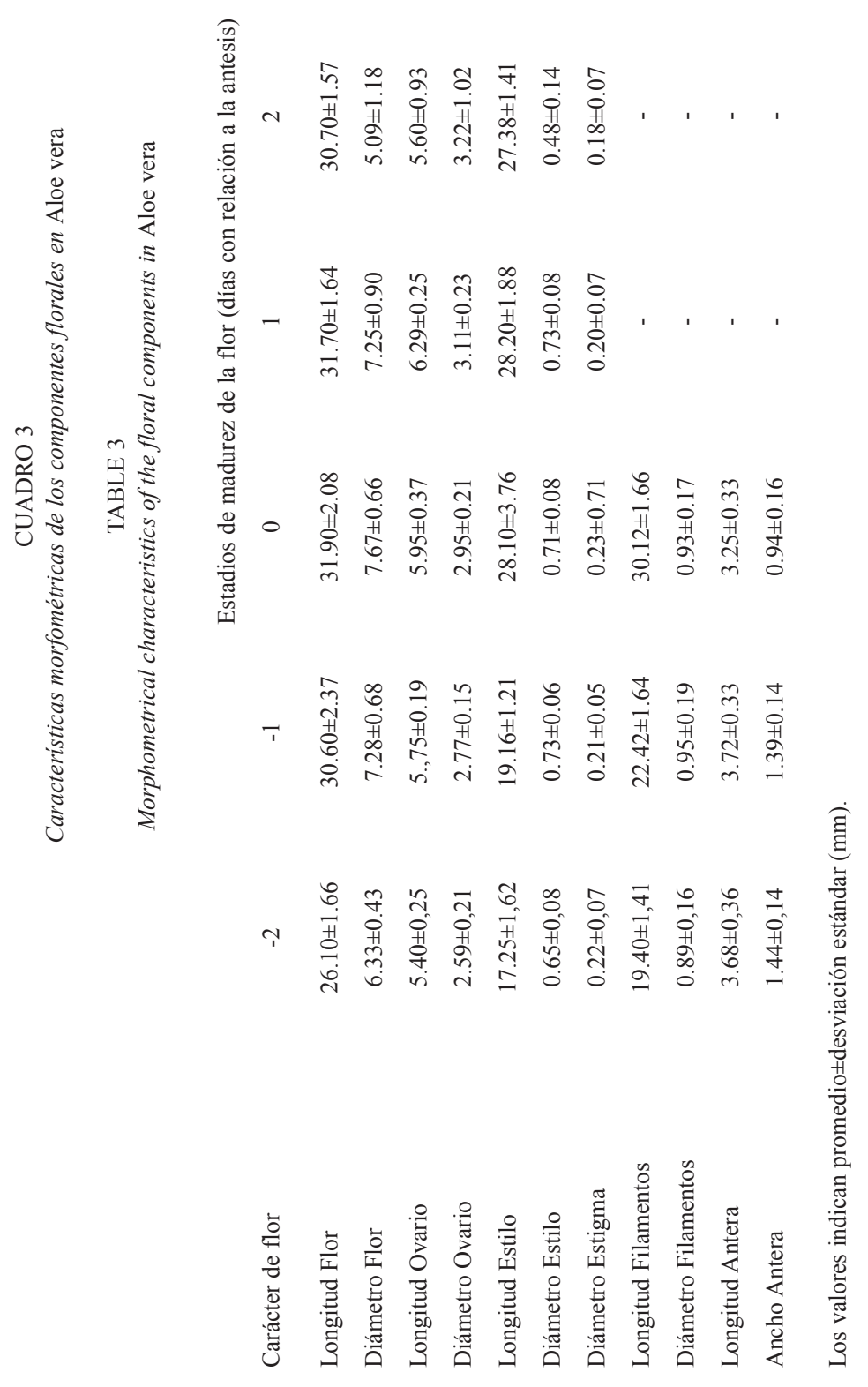




$$
\begin{aligned}
& \text { + }
\end{aligned}
$$

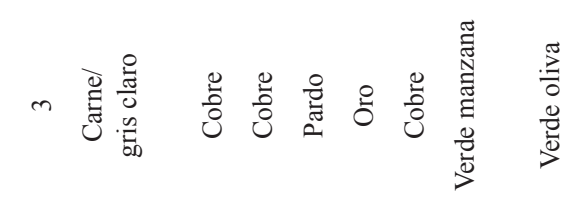

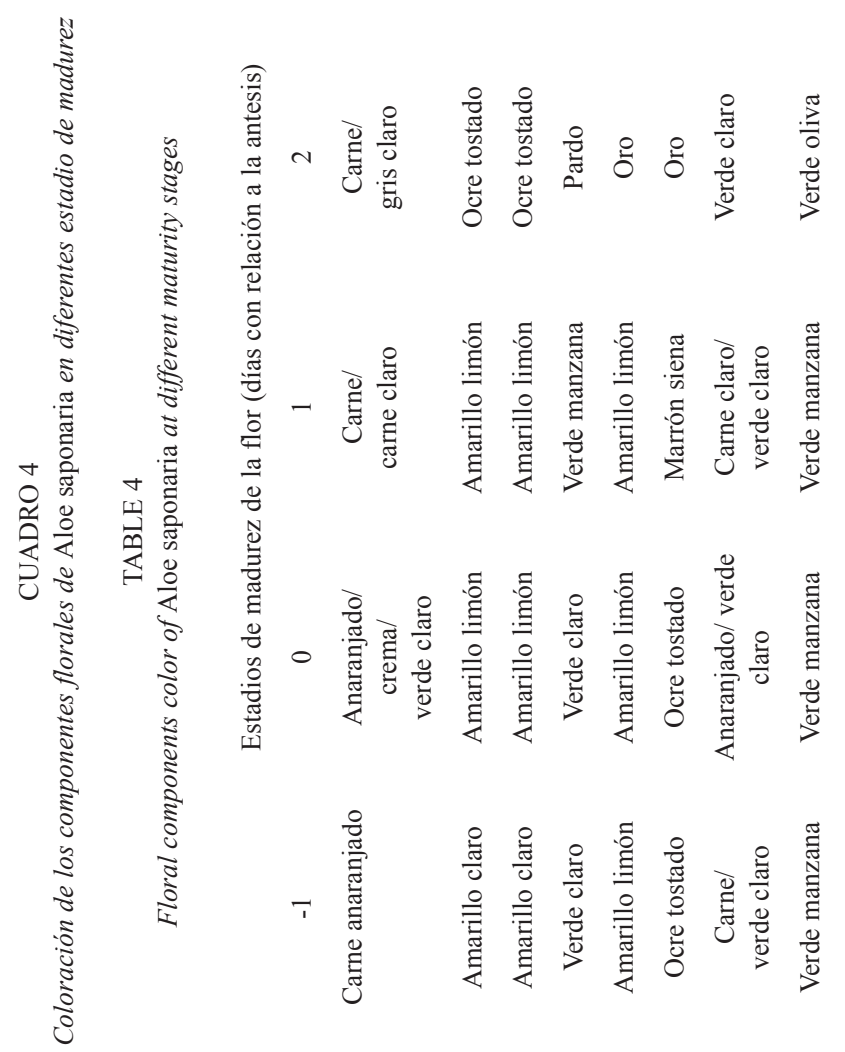

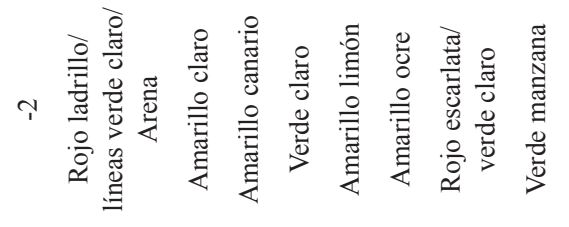

竞 


$$
\text { - ImI!n! }
$$

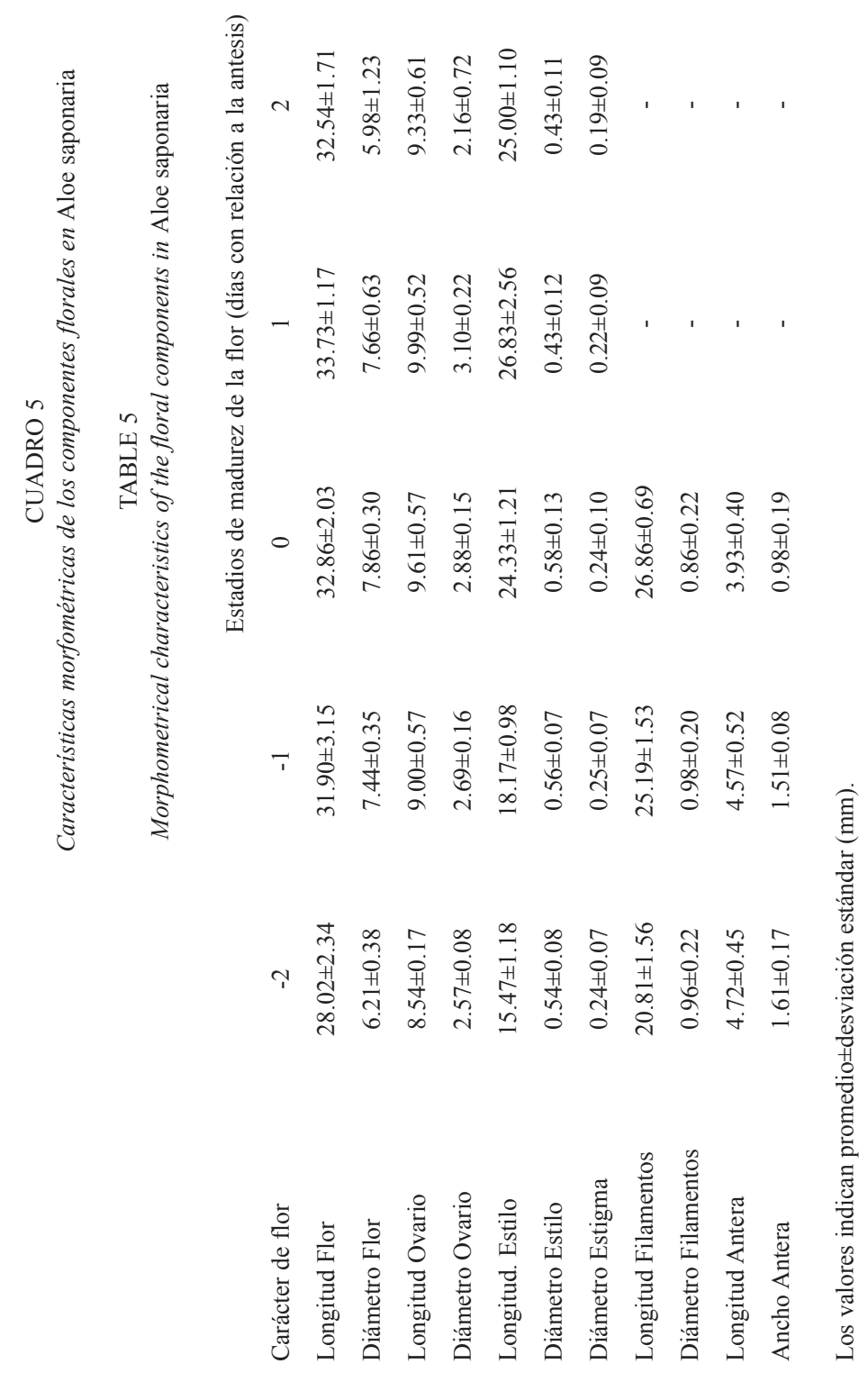


A. vera es de 25-37 mm. En ambas especies el gineceo está conformado por tres carpelos sincárpicos; ovario súpero con forma alargada y oblonga en la base (Fig. 4), trilocular con dos hileras de óvulos por lóculo en placentación axial; estilo simple, cilíndrico, delgado y alargado; estigma pequeño y plumoso.

Receptividad del estigma: en las dos especies el estigma permaneció receptivo durante la antesis y los posteriores estadios de maduración floral, presentando su mayor intensidad un día después de la antesis (Cuadro 6).

Relación Polen/Óvulo (P/O): el índice $\mathrm{P} / \mathrm{O}$ en $A$. vera resultó 1.28 veces mayor que en $A$. saponaria (Cuadro 7). Los índices obtenidos en estas especies fueron comparados con el sistema reproductivo propuesto por Cruden (1977), y entraron en la categoría de xenogamia obligatoria.

Volumen de néctar y concentración de sacarosa: la presencia de néctar se observó a partir de la antesis y durante los estadios posteriores, siendo mayor la cantidad acumulada de néctar al día después de la antesis (Cuadro 8). En los estadios de maduración restantes, el volumen de néctar cuantificado fue escaso. La concentración de sacarosa en el néctar de A. vera estuvo comprendida entre 13.6-20.9\%; mientras que $A$. saponaria presentó un ámbito de concentración entre 16.2-20.9\%.

\section{CUADRO 6}

Reacción del peróxido de hidrógeno sobre estigmas de Aloe vera y A. saponaria

TABLE 6

Hydrogen peroxide reaction on stigmas of Aloe vera and A. saponaria

\begin{tabular}{lcc} 
& \multicolumn{2}{c}{ Aloe vera y Aloe saponaria } \\
Estadios de madurez de la flor (días con respecto a la antesis) & Reacción & - \\
Dos días antes ( -2 ) & - & - \\
Un día antes $(-1)$ & + & $\mathrm{M}$ \\
El día de antesis ( 0 ) & + & $\mathrm{A}$ \\
Un días después $(+1)$ & + & $\mathrm{M}$ \\
Dos días después $(+2)$ & + & $\mathrm{B}$ \\
Tres días después $(+3)$ & $\mathrm{MB}$ \\
Cuatro días después $(+4)$ & + &
\end{tabular}

CUADRO 7

Relación polen/óvulo en Aloe vera y A. saponaria

TABLE 7

Pollen/ovule ratio in Aloe vera and A. saponaria

$\begin{array}{lccc}\text { Especie } & \begin{array}{c}\text { Número de granos } \\ \text { de polen por flor }\end{array} & \begin{array}{c}\text { Número de óvulos } \\ \text { por flor }\end{array} & \begin{array}{c}\text { Relación } \\ \text { polen/óvulo }\end{array} \\ \text { A. vera } & 249487.12 & 60.1 & 4151.18 \\ \text { A. saponaria } & 331205.22 & 102.0 & 3247.11\end{array}$


A
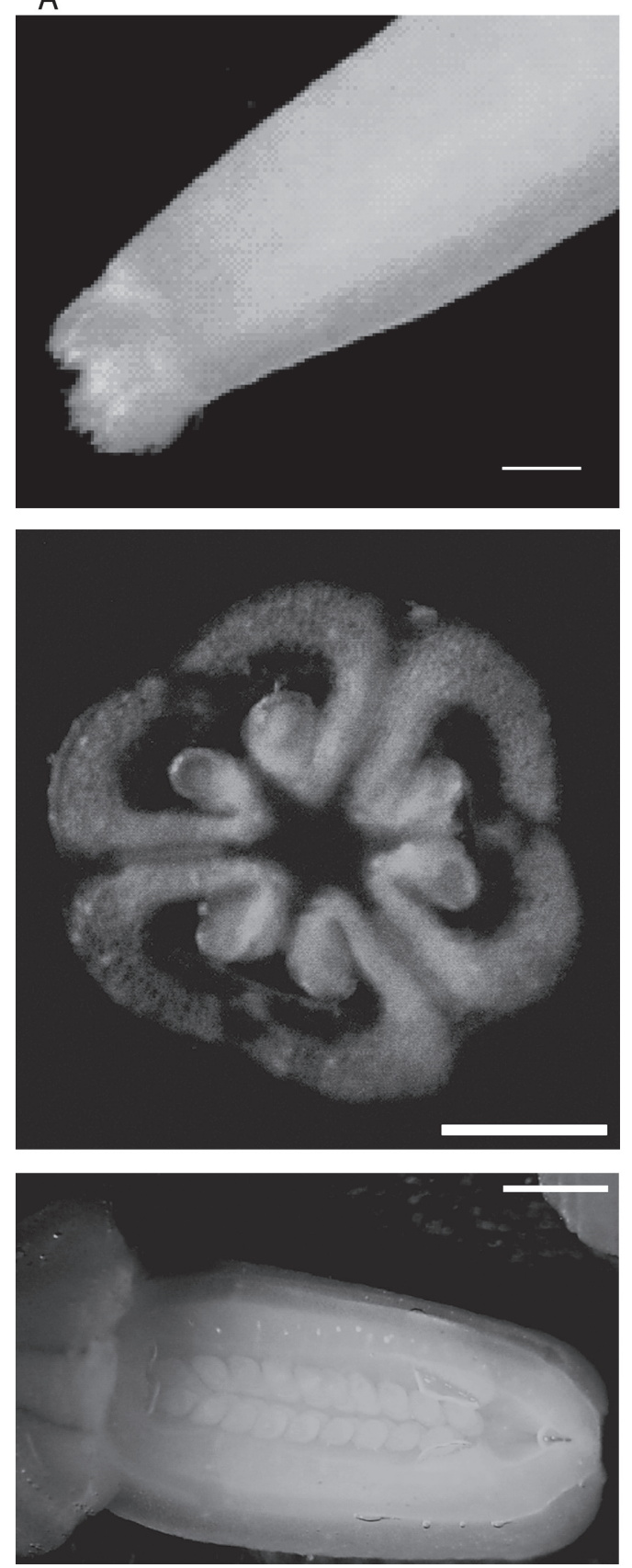

\section{B}
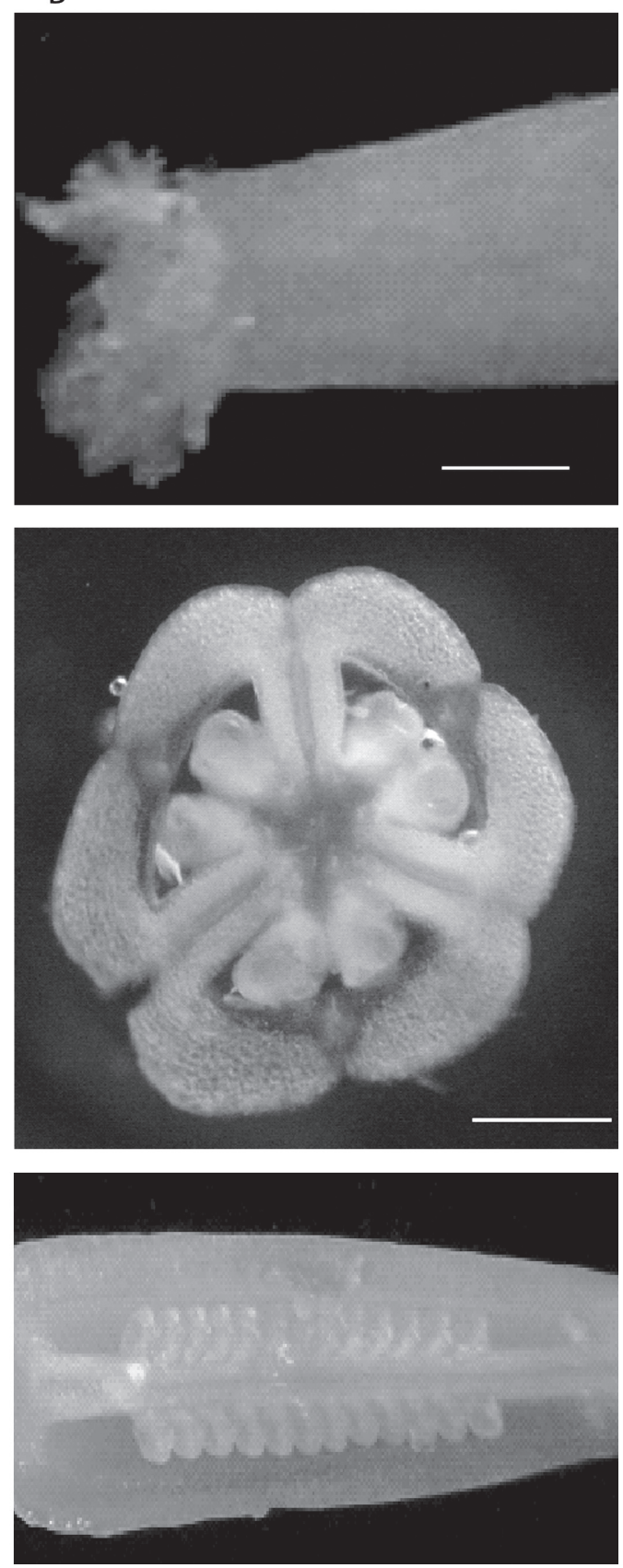

Fig. 4. Rasgos morfoanatómicos del gineceo en flores de Aloe vera (A) y A. saponaria (B). Barra $=1 \mathrm{~mm}$.

Fig. 4. Gynoecium morphoanatomic features in flowers of Aloe vera (A) and A. saponaria (B). Bar $=1 \mathrm{~mm}$. 
CUADRO 8

Volumen de néctar ( $\mathrm{ml}$ ) en flores de Aloe vera $y$ A. saponaria

TABLE 8

Nectar volume $(\mathrm{ml})$ in flowers of Aloe vera and A. saponaria

\begin{tabular}{|c|c|c|c|c|c|c|c|}
\hline \multirow[b]{2}{*}{ Especie } & \multicolumn{7}{|c|}{ Estadios de maduración floral } \\
\hline & -2 & -1 & 0 & 1 & 2 & 3 & 4 \\
\hline A. vera & - & - & 0.30 & 0.37 & 0.07 & 0.06 & 0.05 \\
\hline A. saponaria & - & - & 0.34 & 0.36 & 0.08 & 0.06 & 0.04 \\
\hline
\end{tabular}

CUADRO 9

Visitantes florales en Aloe vera $y$ A. saponaria

TABLE 9

Floral visitors to Aloe vera and A. saponaria

$\begin{array}{lc}\text { Visitante floral } & \text { Familia } \\ \text { Apis mellifera } & \text { Apidae } \\ \text { Trigona } \text { sp. } & \text { Meliponidae } \\ \text { Poliste } \text { sp. } & \text { Vespidae } \\ \text { Eumenes sp. } & \text { Vespidae } \\ \text { Vespa } \text { sp. } & \text { Vespidae } \\ \text { Leucippus fallax } & \text { Trochilidae } \\ \text { Amazilia tobaci } & \text { Trochilidae }\end{array}$

Frecuencia

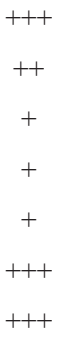

Frecuencia: $(+++)$ alta, $(++)$ media, $(+)$ baja.

Visitantes florales: las flores de $A$. vera y $A$. saponaria fueron visitadas por cinco especies de insectos pertenecientes al orden Himenóptera, distribuidas en las familias Apidae, Meliponidae, Vespidae y dos especies de aves pertenecientes al orden Apodiforme de la familia Trochilidae. La tasa de visita de estas especies para recolectar néctar y polen fue mayor en la mañana (Cuadro 9) y la especie que presentó mayor número de individuos visitantes fue Apis mellifera.

\section{DISCUSIÓN}

Las plantas de Aloe vera cultivadas en condiciones de vivero iniciaron su floración en diciembre, similar a lo observado por Imery y Cequea (2002) en poblaciones naturalizadas de la península de Araya. En ambos ambientes, el inicio de la floración coincide con la temporada anual en la cual disminuyen el fotoperíodo y la temperatura media diaria, hasta promedios de $11.5 \mathrm{~h}$ luz y $23^{\circ} \mathrm{C}$, respectivamente (Guevara et al. 1992). No obstante, en las condiciones experimentales y etapa de evaluación de la presente investigación, la floración culminó un mes después (abril) de lo señalado por los mencionados autores.

Las plantas de $A$. saponaria presentaron un período de floración más amplio que las de $A$. vera, el cual estuvo comprendido entre noviembre y agosto. Esto concuerda con lo indicado por Van-Wyk y Smith (1996), quienes señalan que el período de floración de $A$. saponaria es muy variado con relación a otras especies de Aloe, y puede extenderse dependiendo 
de la zona en que se encuentre. No obstante, se evidenció un notable solapamiento en los períodos de floración de ambas especies, lo cual resulta conveniente para la planificación de cruzamientos interespecíficos de utilidad en programas de mejora genética de rasgos agronómicamente importantes o para la obtención de híbridos de valor ornamental.

Las dos especies presentaron inflorescencias en forma de racimo simple o compuesto sobre un escapo erguido de aproximadamente $90 \mathrm{~cm}$ de longitud para $A$. vera, tal como lo mencionan Hoyos (1985), Sanabria (1978), Schnee (1984) y Rosaspini (1996); y sobre un escapo ondulado de $70 \mathrm{~cm}$ para $A$. saponaria, más largo que lo indicado por Hoyos (1982). Estas formas de escapo han sido descritas con anterioridad por otros autores en plantas pertenecientes a este género (Carter 1994).

A pesar de que las dos especies presentaron homogeneidad en cuanto a los caracteres morfométricos de las flores, todos los valores de longitud, tanto del perianto como de los componentes del androceo y del gineceo en cada especie, son superiores a los señalados por Hoyos (1982, 1985); Schnee (1984) y Rosaspini (1996).

La placentación axial de los óvulos en las especies estudiadas, coincide con la descrita por Cronquist (1981), pero no con la de Carter (1994), que indica una placentación central en el género Aloe, clasificación que es errónea, considerando que este tipo de placentación presume la existencia de un ovario unilocular ${ }^{1}$, por lo cual es posible que se trate de alguna confusión o uso incorrecto de los términos botánicos, a razón de que las especies estudiadas son claramente triloculares.

La mayoría de las características de la inflorescencia de $A$. vera presentaron mayores dimensiones que las de $A$. saponaria. Es posible que la mayor biomasa vegetativa de $A$. vera, permita desarrollar un escapo más robusto con

1. Ivelisse Guevara. Profesora de Anatomía Vegetal, Departamento de Biología, Universidad de Oriente. (Comunicación personal). un mayor número de flores y a su vez presentar una mayor duración de la floración. También es de hacer notar que la menor longitud efectiva de la inflorescencia de A. saponaria es una consecuencia del tipo de racimo compacto, en donde la separación entre los nudos florales es muy estrecha.

Las dos especies mostraron una clara sincronización entre la apertura de la flor y la dehiscencia de las anteras. Este suceso también fue observado por Nassar et al. (1997) y Viana et al. (2001) en cactáceas columnares como Stenocereus griseus, Pilosocerus moritizianus, Subpilocerus repandus, S. horripinis y Trichocereus pasacana. Por otra parte, en ambas especies de Aloe, se determinó que el estigma se encontraba más receptivo el día después de la dehiscencia de las anteras, lo que indica que estas especies presentan dicogamia del tipo protandria, debido a que las anteras de la flor liberan los granos de polen al menos $24 \mathrm{~h}$ antes del periodo de mayor receptividad de su estigma, resultados que concuerdan con lo descrito por Imery (2001), con base en la frecuencia de formación de frutos y semillas luego de cruzamientos dirigidos con polen de A. saponaria sobre estigmas de $A$. vera en diferentes estadios de maduración de la flor receptora. Pailler y Warren (2002), también describen la presencia de este tipo de dicogamia en la especie A. mayottensis. Este desfase temporal, constituye una estrategia para captar el polen transferido de otras flores, lo cual favorece la polinización cruzada (Webb 1985; Hoc y García 1998).

La dicogamia en ambas especies de Aloe es asincrónica, al igual que en plantas del género Retanilla (Medan y Arce 1999), debido a que las flores no abren al mismo tiempo, sino por nudos, permitiendo a los visitantes florales encontrar flores en varios estadios de maduración floral en la misma inflorescencia, haciendo posible la autopolinización. Esto sugiere la existencia de una barrera reproductiva complementaria que limitase la autogamia en grandes poblaciones de una misma especie de Aloe.

La producción promedio de néctar en las flores de ambas especies de Aloe ( $0.34 \mathrm{ml} /$ flor) 
fue parecida a la informada por Mione y Serazo (2001) en Jaltomata biflora ( $0.40 \mathrm{ml} / \mathrm{flor})$ y superior a las observadas en Trichocereus pasacana $(0.128 \mathrm{ml} /$ flor) de Argentina (Viana et al. 2001), plantas con flores cuatro veces mayor al tamaño de las flores de Aloe. Este volumen de néctar también resultó superior a lo registrado en Phaseolus vulgaris con un promedio acumulado de $0.18 \mathrm{ml} /$ flor durante toda la antesis (Hoc y Garcia 1998); Kalanchoe pinnata $(0.012 \mathrm{ml} /$ flor), $K$. daigrernontiana $(0.065 \mathrm{ml} /$ flor $)$ y en Eritrina mitis $(0.039 \mathrm{ml} /$ flor) (Grasses y Ramírez 1998). Sin embargo, la concentración de sacarosa en el néctar de las especies estudiadas (13-21\%) fue inferior a la de estas especies, como es el caso de T. pasacana (20-38\%) (Viana et al. 2001), P. vulgaris (30.9\%) (Hoc y Garcia 1998), Passiflora foetida (34\%) (García y Hoc 1995) o las plantas de $J$. biflora con una concentración de sacarosa entre 14.2-57.6\% (Mione y Serazo 2001).

El promedio de néctar por inflorescencia en $A$. vera es de $77.6 \mathrm{ml}$ y en $A$. saponaria es de $31.8 \mathrm{ml}$, y cada una de estas plantas puede llegar a formar hasta tres inflorescencias durante la época de floración, triplicando así el volumen de néctar disponible. Esta considerable cantidad de néctar, aunada a la elevada frecuencia de visitas por $A$. mellifera, permiten suponer que ambas especies de Aloe poseen un importante valor en la apicultura. Este argumento coincide con lo referido por Newton (2004), en base a los cambios de conducta adoptados por las abejas y la mayor producción de miel observada durante la floración de las especies de Aloe en poblaciones naturales de Sudáfrica.

La coloración es considerada como uno de los rasgos involucrados en la elección de las flores por parte de los polinizadores (Proctor y Yeo 1979). La frecuente visita de varios insectos y aves en las especies estudiadas tiene mucho que ver con la coloración del perianto y otros componentes florales (androceo y gineceo), lo cual en conjunto con el tipo de inflorescencia racemosa, capaz de producir un gran número de flores, permite que las inflorescencias de estas plantas sean sensorialmente llamativas para muchos visitantes florales.
Los colores amarrillo, verde, rojo y anaranjado, presentes en las flores de $A$. vera y $A$. saponaria, son considerados por distintos autores como capaces de atraer a una gran diversidad de agentes polinizadores. Xena y Madriz (1994), al igual que Ramírez (1997), consideran al color amarrillo como el segundo en la escala de colores por atraer a un grupo variado de visitantes, seguido de los colores verde, rojo y anaranjado que son visitados por varias especies de animales, pero en menor número. La atracción de estos colores se debe, por ejemplo, en las aves, a que detectan luz en casi todo el espectro ultravioleta, sintiéndose más atraídas por las flores que presentan colores intensos, amarillos, rojos, lo cual es muy notable en los colibríes que se orientan hacia las flores utilizando esta franja del espectro visible (Mizrahi 2003). Por su parte, las abejas tienen una visión que va del azul al espectro ultravioleta, siendo capaces de diferenciar tres colores complementarios entre varias intensidades de gris: el amarillo, el verde-azul y el azul. El espectro visible para las abejas, tiende hacia las radiaciones de longitud de onda corta; el rojo no pueden verlo y fácilmente lo confunden con el negro; en cambio, el ultravioleta, ellas lo ven como un color. Son mucho menos sensibles en el área de los amarillos, pero después de visitar durante 3-4 h pueden llegar a diferenciar el amarillo, el anaranjado y el verde.

Xena y Madriz (1994) y Ramírez (1997) observaron que las flores de igual color a las de $A$. vera y $A$. saponaria, fueron visitadas por dos colibríes, uno de ellos Leucippus fallax, y muchos himenópteros, resaltando la visita de Apis mellifera, al igual que en las plantas estudiadas en este trabajo.

Medan y Arce (1999), en especies de Retanilla, informan algunos rasgos comunes a los de A. vera y A. saponaria, por ejemplo, la elevada producción de polen, una cantidad considerable de néctar con concentración de sacarosa casi similar y flores protándricas dispuestas en racimos. Estas características atrajeron a un gran número de insectos (37 himenópteros en total), siendo una de las especies la abeja ( $A$. mellifera). La diferencia en 
cuanto al mayor o menor número de visitantes florales, está sujeta al sitio en que se encuentran distribuidas las plantas y a la época de floración de cada especie. A pesar de que las dos especies de Aloe compartieron los mismos visitantes florales y en la misma frecuencia de visitas, la especie que presentó mayor densidad de ejemplares visitantes fue $A$. vera. Esto puede deberse a que la inflorescencia en estas plantas presentan un mayor número de flores, lo cual en conjunto ofrece una intensa atracción sensorial para los visitantes florales. Esto explica que la especie visitante con mayor número de ejemplares/inflorescencia sea $A$. mellifera. Además, el solapamiento de los periodos de floración en ambas especies de Aloe, mantenidas en espacios cercanos y bajo las mismas condiciones experimentales, también representa una de las razones elementales para compartir los visitantes florales.

La morfometría de las flores, el color del perianto, la elevada producción de néctar y el tipo de inflorescencia asincrónica en $A$. vera y A. saponaria, son rasgos florales que determinan que el síndrome de polinización de estas plantas sea ornitofílico. Los datos de producción de néctar concuerdan con las observaciones de Percival (1965), quien señala un elevado volumen de néctar, como característica principal de las plantas polinizadas por colibríes.

Por otra parte, no se puede descartar que en estas plantas de Aloe las abejas jueguen un papel importante como polinizadores, ya que el estigma de las flores se expone a partir del segundo día de antesis como consecuencia del alargamiento del estilo y el inicio de la marchitez de los tépalos, permitiendo así que las abejas, cuando van en busca de néctar y polen, entren en contacto directo con el estigma. El síndrome de polinización ornitofílico ha sido sugerido también por Cronquist (1981) para la mayoría de las especies de género Aloe que presentan flores de tamaño igual o superior a las de A. vera y A. saponaria. En este sentido, la existencia de más de un síndrome de polinización no es extraño, como lo señalan Xena y Madriz (1994) y Ramírez (1997), debido a que existen especies vegetales que presentan inflorescencias de colores muy llamativos para un gran grupo de visitantes florales que actúan como polinizadores.

La baja producción de frutos en $A$. saponaria y la ausencia total de estos en $A$. vera, a pesar de la gran producción de flores, se podría atribuir erróneamente a la escasez de especies polinizadoras; sin embargo, la frecuente visita de varias especies de insectos y de pequeños pájaros durante el período de antesis y la observación de estigmas impregnados de granos de polen, ponen de manifiesto una intensa y efectiva polinización, actividad que también fue observada por Imery y Cequea (2002), en una extensa población de $A$. vera ubicada en el oriente de Venezuela.

La ausencia de frutos en $A$. vera sugiere que, además de la protandría, existe un mecanismo complementario de autoincompatibilidad que limita la reproducción sexual en esta especie. La autoincompatibilidad en algunas especies de género Aloe fue propuesta inicialmente por Berger (1908), citado por Riley y Majumdar (1979) al observar que las plantas de A. aethiopia, A. pluridens y A. caesia no formaban frutos luego de una intensa autopolinización. Los altos valores de la relación $\mathrm{P} / \mathrm{O}$ determinados en $A$. vera, contribuyen con esta hipótesis, puesto que es característico de las plantas autoincompatibles.

Los sistemas de apareamiento no fueron evaluados en esta investigación, debido a que habían sido estudiados con anterioridad y reportadas por Imery (2001). Este autor practicó numerosas polinizaciones controladas intra e interespecíficas con ejemplares conservados en vivero, observando formación de frutos sólo cuando las flores de A. saponaria eran autopolinizadas, o en aquellos cruzamientos en los que $A$. vera actuaba como progenitor femenino. Las flores autopolinizadas de $A$. vera, y las de A. saponaria que actuaron como receptoras del polen de $A$. vera, no desarrollaron frutos. Al colocar barreras artificiales sobre las inflorescencias, limitando así el acceso de los polinizadores, los frutos estuvieron ausentes en ambas especies. Los resultados de las polinizaciones artificiales y las evaluaciones de la eficiencia 
reproductiva en condiciones naturales, en base a la relación entre el número de flores y frutos, sustentan la existencia de un mecanismo de autoincompatibilidad en ambas especies, el cual reduce la cantidad de frutos potenciales en A. saponaria y limita completamente su formación en $A$. vera.

Los trabajos de Riley y Majumdar (1979) e Imery (2001) refieren la existencia de autoincompatibilidad esporofítica en especies de la familia Aloaceae. En A. vera, esta barrera natural para la autofertilización, en combinación con la escasa variabilidad, como consecuencia de la exclusiva propagación vegetativa y la existencia de anormalidades cromosómicas durante la formación del polen (Sapre 1975; Imery y Cequea 2002), disminuyen las posibilidades de que los granos de polen normales germinen sobre estigmas de flores de la misma especie, reduciendo así las expectativas de reproducción sexual en esta especie.

\section{AGRADECIMIENTOS}

Los autores agradecen a José Véliz por prestar algunos equipos, a Pablo Cornejo y Jorge Muñoz por la identificación de visitantes florales, a William Lampe por la asesoría en aspectos de microscopía y fotografía y al Concejo de Investigación de la Universidad de Oriente (Venezuela) por el financiamiento a través del proyecto CI-5-010101-1223/05.

\section{RESUMEN}

Se analizó la fenología reproductiva y aspectos anatómicos florales en dos especies de Aloe conservadas en condiciones de vivero en el oriente de Venezuela, de septiembre 2001 a septiembre 2002. A. vera floreció de diciembre a mayo; A. saponaria de noviembre a agosto. En ambas especies, la antesis duró 48 h, la dehiscencia de la antera coincidió con la apertura del perianto, la receptividad del estigma fue mayor al segundo días de antesis y la producción de néctar alcanzó $0.34 \mathrm{ml} /$ flor. En $A$. vera se observaron $228 \pm 77$ flores de color amarillo sobre escapos extensos de $76 \pm 11 \mathrm{~cm}$ y 1-3 ejes; a diferencia de A. saponaria con $94 \pm 33$ flores anaranjadas en escapos más compactos de $62 \pm 5 \mathrm{~cm}$ y 3-5 ejes. La relación polen/óvulo fue de 4151.2 en $A$. vera y 3247.1 en $A$. saponaria, clasificándose como xenógamas obligatorias. Se registraron visitas frecuentes de Apis mellifera, Trigona sp., Poliste sp., Eumenes sp., Vespa sp., Leucippus fallax y Amazilia tobaci. A pesar de que ambas especies manifestaron una elevada producción de polen, néctar y la presencia de potenciales polinizadores, al término de la floración, sólo se observaron frutos en $A$. saponaria, con una eficiencia reproductiva de $12 \%$, lo cual, junto a los resultados de la receptividad estigmática y los ensayos preliminares de cruzamientos intra e interespecíficos, sugiere la existencia de protandría y autoincompatibilidad como barreras reproductivas que reducen la endogamia en estas especies.

Palabras claves: Biología reproductiva, polinización, fenología, síndrome floral, melitofilia.

\section{REFERENCIAS}

Carter, S. 1994. Aloaceae, p. 1-60. In R.M. Polhill (ed.). Flora of tropical east Africa. Balkema, Rotterdam, Holanda Meridional, Países Bajos.

Cronquist, A. 1981. An integrated system of classification of flowering plants. Botanical Garden. New York, EE.UU.

Cruden, R. 1977. Pollen-ovule ratios: A conservative indicator of breeding systems in flowering plants. Evolution 31: 32-46.

García, M. \& P. Hoc. 1995. Biología floral de Passiflora foetida (Passifloraceae). Rev. Fac. Agro. 13: 190201.

Grasses, C. \& N. Ramírez. 1998. Biología reproductiva de cinco especies ornitófilas en un fragmento de bosque caducifolio secundario en Venezuela. Rev. Biol. Trop. 46.

Guevara, M., Y. Bergerón., R. McNeil \& A. Leduc. 1992. Seasonal flowering and fruiting in tropical semi-arid vegetation of northeastern Venezuela. Biotropica 24: 64-76.

Hoc, P. \& M. García. 1998. Biología floral y sistema reproductivo de Phaseolus vulgaris var. aborigineous. Rev. Fac. Agro. 14: 176-188.

Hoyos, J. 1982. Guía de los árboles de Venezuela I. Soc. Cienc. Nat. La Salle. Monografía No 31. Caracas, Venezuela.

Hoyos, J. 1985. Flora de la isla de Margarita, Venezuela. Soc. Ciencias Nat. La Salle, Caracas, Venezuela.

Imery, J. 2001. Protandría y autoincompatibilidad en Aloe vera L. LI AsoVAC. San Cristobal, Venezuela. Acta Cient. Venez. 55: s.p. 
Imery, J. \& H. Cequea. 2002. Anormalidades cromosómicas en la microsporogénesis de Aloe vera (L.) Burm. f. (Aloaceae). Acta Bot. Venez. 25: 143-152.

Medan, D. \& M. Arce. 1999. Reproductive biology of the Andean-disjunct genus Retanilla (Rhamnaceae). Plant Syst. Evol. 218: 281-291.

Mione, T. \& A. Serazo. 2001. Rediscovery and flora biology of Jaltomata biflora (Solonaceae). Econ. Bot 55: 167-170.

Mizrahi, D. 2003. La visión de las aves. Zeledonia 7: 19.

Nassar, J., N. Ramírez \& O. Linares. 1997. Comparative pollination biology of venezuelan columnar cacti and the role of nectar-feeding bats in their sexual reproduction. Amer. J. Bot. 84: 918-927.

Newton, L.E. 2004. Aloe in habitat, p. 3-14. In T. Reynolds (ed.). Aloes, the genus Aloe. CRC Press, Boca Ratón, Florida, EE.UU.

Osborn, M., P. Kevan \& M. Lane. 1988. Pollination biology of Opuntia polyacantha and Opuntia plaeacantha (Cactaceae) in southern Colorado. Plant Syst. Evol. 159: 85-94.

Pailler, T. \& B. Warren. 2002. Biologie de la reproduction de Aloe mayottensis (Liliaceae), une espece endemique de l'ile Mayotte (Ocean indien). Can. J. Bot. 80: 340-348.

Percival, M. 1965. Flora biology. Pergamon, Londres, Inglaterra.

Proctor, M. \& P. Yeo. 1979. The pollination of flowers. Collins, Londres, Inglaterra.

Ramírez, N. 1997. Biología reproductiva y selección de especies nativas para la recuperación de áreas degradadas: métodos y significado. Acta Bot. Venez. 20: 43-66.
Riley, H.P. \& S.K. Majumdar. 1979. The Aloineae: a biosystematic survey. University Press, Lexington, Kentucky, EE.UU.

Rosaspini R. 1996. Aloe vera, una planta milagrosa. Equipo de Investigación Nueva Era. Edic. Continente. Buenos Aires, Argentina.

Sapre, A. 1975. Meiosis and pollen mitosis in Aloe barbadensis Mill. (A. perfoliata var. vera L. A. vera Auth. non Mill.). Cytologia 40: 525-533.

Sanabria, M. 1978. Estudio sistemático de manglares de la Región Nor-Oriental Venezuela. Trabajo de pregrado. Departamento de Biología, Universidad de Oriente, Cumaná, Venezuela.

Schnee, L. 1984. Plantas comunes de Venezuela. $3^{\text {ra }}$ Edic. Universidad Central de Venezuela. Ediciones de la Biblioteca, Caracas, Venezuela.

Smith, G. \& B. Van-Wyk. 1991. Generic relations chips in the Aloideae (Asphodelaceae). Taxon 40: 557-581.

Van-Wyk, B. \& G. Smith. 1996. Guide to the Aloes of South Africa. Briza Publ., Pretoria. Sudáfrica.

Viana, M., P. Baes., M. Saravia., E. Badano, \& B. Schulumpberger. 2001. Biología floral y polinizadores de Trichocereus pasacana (Cactaceae) en el Parque Nacional Los Cardones, Argentina. Rev. Biol. Trop. 29: 277-283.

Webb, C. 1985. Protandry, pollination, and self-incompatibility in Discaria toumatou, New Zealand. J. Bot. 23: 331-335.

Xena, N. \& R. Madriz. 1994. Aspectos de la biología de polinización en el bosque enano de la cima del "Cerro Copey” (Isla Margarita). Acta Bot. Venez. 17: 35-68.

\section{REFERENCIA DE INTERNET}

Sánchez, J. 1999. Los aloes. Edic. On line: http://www. busca-plantas.com/Abe.htm (consultado 10 febrero 2005). 
